\title{
Acoustic wave scattering from transversely isotropic cylinders
}

\author{
F. Honarvar and A. N. Sinclair \\ Department of Mechanical Engineering, University of Toronto, 5 King's College Road, Toronto, \\ Ontario M5S 1A4, Canada
}

(Received 16 October 1995; accepted for publication 14 March 1996)

\begin{abstract}
Mathematical expressions are derived for the far-field backscattering amplitude spectrum resulting from oblique insonification of an infinite, transversely isotropic elastic cylinder by a plane acoustic wave. The normal-mode solution is based on decoupling of the scalar potential representing the horizontally polarized shear wave from those of the compressional and vertically polarized waves. The solution degenerates to the well-known simple model for isotropic cylinders in the case of very weak anisotropy. The solution is used to study the influence of each element of the stiffness matrix on the various resonant modes of vibration. Perturbations of the elements $c_{33}$ and $c_{44}$, which characterize the cylinder along the axis, significantly affect resonant frequencies corresponding to axially guided waves. Perturbations of $c_{11}$ and $c_{12}$, which characterize the material on the transverse plane, predominantly affect the Rayleigh and Whispering Gallery resonance frequencies. Perturbations of $c_{13}$ affect all three types of resonances. These results are consistent with elasticity theory and the known modal shapes of these resonances. (c) 1996 Acoustical Society of America.

PACS numbers: 43.20.Bi, 43.20.Fn, 43.20.Ks [WGN]
\end{abstract}

\section{INTRODUCTION}

One possible application of resonance acoustic scattering is in nondestructive evaluation (NDE) of cylindrical components. This is achieved by studying the perturbations in the resonant frequencies of the backscattered amplitude spectrum, and correlating these perturbations to changes in certain properties of the cylinder.

Previous studies in resonance acoustic scattering are mostly concerned with isotropic cylinders and shells. In the NDE of cylindrical components, such as wires, rods, and pipes, the sample is usually transversely isotropic due to the processes used in manufacturing these products. Transverse isotropy is usually desirable, since it provides higher strength/stiffness along the cylinder axis. This paper discusses the development of a mathematical model for acoustic wave scattering from such transversely isotropic cylinders.

Numerous works on acoustic wave scattering from isotropic cylinders and shells have appeared in the literature during the past few years. The first, pioneering study of acoustic wave scattering from submerged solid elastic cylinders based on a normal-mode expansion is due to Faran. ${ }^{1}$ The more general problem, when the propagation direction of the incident wave makes an arbitrary angle $\alpha$ with the normal to the cylinder, was considered by Flax et al. ${ }^{2}$ The similar problem for a cylindrical shell was studied by Léon et $a l^{3}$ and Veksler. ${ }^{4}$

Theoretical studies on wave propagation in anisotropic cylinders and shells have also been pursued for many years. Mirsky $^{5,6}$ has studied the propagation of free harmonic waves in transversely isotropic circular cylinders. Tsai et al. ${ }^{7}$ and $\mathrm{Tsai}^{8}$ investigated the cylindrically guided waves in transversely isotropic shafts and thick hollow cylinders.

Theoretical works are also available for propagation of acoustic waves in cylindrical boreholes surrounded by anisotropic media. White and Tongtaw ${ }^{9}$ developed a mathematical model for elastic wave propagation in a cylindrical, fluid- filled borehole in a transversely isotropic solid. Chan and Tsang ${ }^{10}$ extended this problem to the propagation of acoustic waves in a fluid-filled borehole surrounded by concentrically layered, transversely isotropic media. Kundu and Boström ${ }^{11}$ studied the scattering of a plane wave by a circular crack in a transversely isotropic solid. Zhang et al. ${ }^{12}$ analyzed the acoustic field excited by multipole sources in a fluid-filled borehole surrounded by a transversely isotropic medium. The procedures followed in these related works have been considered in formulating the problem at hand.

Quite recently, some purely experimental results on acoustic wave scattering from anisotropic cylinders were reported by de Billy. ${ }^{13,14}$ To the best of our knowledge, no theoretical work on acoustic wave scattering from anisotropic cylinders has appeared in the literature.

\section{FORMULATION OF THE PROBLEM}

In the mathematical model, an infinite plane acoustic wave of frequency $\omega / 2 \pi$ incident at an angle $\alpha$ on a submerged transversely isotropic circular cylinder of infinite length and outer radius $a$ is considered, Fig. 1. A cylindrical coordinate system $(r, \theta, z)$ is chosen with the $z$ direction coincident with the axis of the cylinder. The pressure $p_{i}$ of the incident plane wave external to the cylinder at a point $M(r, \theta, z)$ is represented by ${ }^{1}$

$$
p_{i}=P_{0} \sum_{n=0}^{\infty} \epsilon_{n} i^{n} J_{n}\left(k_{\perp} r\right) \cos n \theta e^{i\left(k_{z} z-\omega t\right)},
$$

where

$$
k_{z}=k \sin \alpha, \quad k_{\perp}=k \cos \alpha,
$$

and $k=\omega c, c$ is the compressional wave velocity in the liquid medium outside the cylinder, $\epsilon_{n}$ is the Neumann factor $\left(\epsilon_{n}=1\right.$ for $n=0$, and $\epsilon_{n}=2$ for $\left.n>0\right), P_{0}$ is the incident pressure wave amplitude, and $J_{n}$ is the Bessel function of the first kind of order $n$. The outgoing scattered wave pressure, $p_{s}$, at 


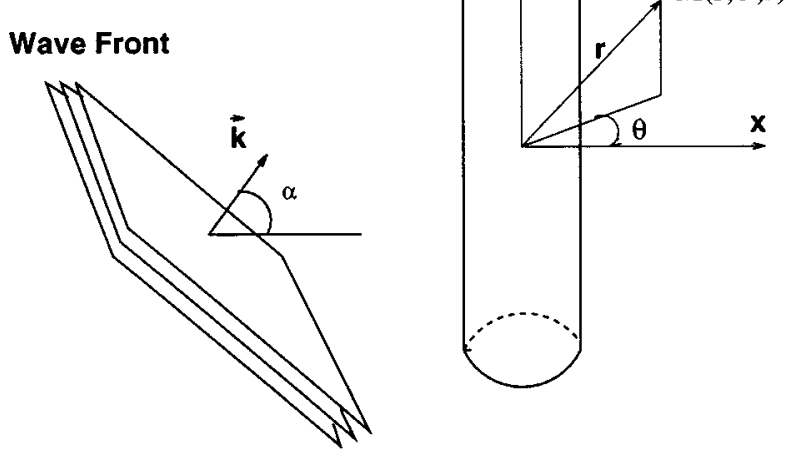

FIG. 1. Plane wave insonification of submerged cylinders.

point $M$ must be symmetrical about $\theta=0$ and, therefore, of the form,

$$
p_{s}=P_{0} \sum_{n=0}^{\infty} \epsilon_{n} i^{n} A_{n} H_{n}^{(1)}\left(k_{\perp} r\right) \cos n \theta e^{i\left(k_{z} z-\omega t\right)},
$$

where $H_{n}^{(1)}$ is the Hankel function of the first kind of order $n$, and $A_{n}$ are the unknown scattering coefficients.

The general Hooke's law for a transversely isotropic material is

$$
\begin{aligned}
\left\{\begin{array}{c}
\sigma_{r r} \\
\sigma_{\theta \theta} \\
\sigma_{z z} \\
\sigma_{\theta z} \\
\sigma_{r z} \\
\sigma_{r \theta}
\end{array}\right\}= & {\left[\begin{array}{cccccc}
c_{11} & c_{12} & c_{13} & 0 & 0 & 0 \\
c_{12} & c_{11} & c_{13} & 0 & 0 & 0 \\
c_{13} & c_{13} & c_{33} & 0 & 0 & 0 \\
0 & 0 & 0 & c_{44} & 0 & 0 \\
0 & 0 & 0 & 0 & c_{44} & 0 \\
0 & 0 & 0 & 0 & 0 & \left(c_{11}-c_{12}\right) / 2
\end{array}\right] } \\
& \times\left\{\begin{array}{c}
\epsilon_{r r} \\
\epsilon_{\theta \theta} \\
\epsilon_{z z} \\
2 \epsilon_{\theta z} \\
2 \epsilon_{r z} \\
2 \epsilon_{r \theta}
\end{array}\right\},
\end{aligned}
$$

where $\sigma_{i j}$ are stress components, $\epsilon_{i j}$ are strain components, and $c_{i j}$ are the elastic constants. A transversely isotropic material is characterized by five independent elastic constants.

In a continuum, equations of motion in the absence of body forces are ${ }^{15}$

$$
\begin{aligned}
& \frac{\partial \sigma_{r r}}{\partial r}+\frac{1}{r} \frac{\partial \sigma_{r \theta}}{\partial \theta}+\frac{\partial \sigma_{r z}}{\partial z}+\frac{1}{r}\left(\sigma_{r r}-\sigma_{\theta \theta}\right)=\rho_{c} \frac{\partial^{2} U_{r}}{\partial t^{2}}, \\
& \frac{\partial \sigma_{r \theta}}{\partial r}+\frac{1}{r} \frac{\partial \sigma_{\theta \theta}}{\partial \theta}+\frac{2}{r} \sigma_{r \theta}+\frac{\partial \sigma_{z \theta}}{\partial z}=\rho_{c} \frac{\partial^{2} U_{\theta}}{\partial t^{2}}, \\
& \frac{\partial \sigma_{r z}}{\partial r}+\frac{1}{r} \frac{\partial \sigma_{\theta z}}{\partial \theta}+\frac{1}{r} \sigma_{r z}+\frac{\partial \sigma_{z z}}{\partial z}=\rho_{c} \frac{\partial^{2} U_{z}}{\partial t^{2}},
\end{aligned}
$$

where $\rho_{c}$ is the cylinder density and $U_{r}, U_{\theta}$, and $U_{z}$ are the displacements in the $r, \theta$, and $z$ directions, respectively. Substituting Eq. (4) in Eqs. (5)-(7) gives

$$
\begin{aligned}
& c_{44}\left(\frac{\partial^{2} U_{z}}{\partial z \partial r}+\frac{\partial^{2} U_{r}}{\partial z^{2}}\right)+c_{11}\left(\frac{\partial^{2} U_{r}}{\partial r^{2}}+\frac{1}{r} \frac{\partial U_{r}}{\partial r}-\frac{3}{2 r^{2}} \frac{\partial U_{\theta}}{\partial \theta}\right. \\
& \left.+\frac{1}{2 r^{2}} \frac{\partial^{2} U_{r}}{\partial \theta^{2}}-\frac{U_{r}}{r^{2}}+\frac{1}{2 r} \frac{\partial^{2} U_{\theta}}{\partial r \partial \theta}\right) \\
& \quad+\frac{1}{2 r} c_{12}\left[\frac{\partial}{\partial \theta}\left(\frac{\partial U_{\theta}}{\partial r}-\frac{1}{r} \frac{\partial U_{r}}{\partial \theta}+\frac{U_{\theta}}{r}\right)\right]+c_{13} \frac{\partial^{2} U_{z}}{\partial z \partial r} \\
& =\rho_{c} \frac{\partial^{2} U_{r}}{\partial t^{2}},
\end{aligned}
$$

$$
\begin{aligned}
c_{44}\left[\frac{\partial}{\partial z}\left(\frac{\partial U_{\theta}}{\partial z}+\frac{1}{r} \frac{\partial U_{z}}{\partial \theta}\right)\right]+c_{12}\left(-\frac{1}{2 r} \frac{\partial U_{\theta}}{\partial r}+\frac{1}{2} \frac{U_{\theta}}{r^{2}}-\frac{1}{2} \frac{\partial^{2} U_{\theta}}{\partial r^{2}}\right. \\
\left.-\frac{1}{2 r^{2}} \frac{\partial U_{r}}{\partial \theta}+\frac{1}{2 r} \frac{\partial^{2} U_{r}}{\partial \theta \partial r}\right)+c_{11}\left(\frac{1}{r^{2}} \frac{\partial^{2} U_{\theta}}{\partial \theta^{2}}+\frac{1}{2 r} \frac{\partial U_{\theta}}{\partial r}\right. \\
\left.+\frac{1}{2} \frac{\partial^{2} U_{\theta}}{\partial r^{2}}+\frac{3}{2 r^{2}} \frac{\partial U_{r}}{\partial \theta}+\frac{1}{2 r} \frac{\partial^{2} U_{r}}{\partial r \partial \theta}-\frac{1}{2} \frac{U_{\theta}}{r^{2}}\right) \\
+\frac{c_{13}}{r} \frac{\partial^{2} U_{z}}{\partial z \partial \theta}=\rho_{c} \frac{\partial^{2} U_{\theta}}{\partial t^{2}}
\end{aligned}
$$

$$
\begin{array}{r}
c_{44}\left(\frac{1}{r^{2}} \frac{\partial^{2} U_{z}}{\partial \theta^{2}}+\frac{\partial^{2} U_{z}}{\partial r^{2}}+\frac{1}{r} \frac{\partial^{2} U_{\theta}}{\partial z \partial \theta}+\frac{1}{r} \frac{\partial U_{z}}{\partial r}+\frac{1}{r} \frac{\partial U_{r}}{\partial z}+\frac{\partial^{2} U_{r}}{\partial z \partial r}\right) \\
+c_{13}\left[\frac{\partial}{\partial z}\left(\frac{U_{r}}{r}+\frac{1}{r} \frac{\partial U_{\theta}}{\partial \theta}+\frac{\partial U_{r}}{\partial r}\right)\right]+c_{33} \frac{\partial^{2} U_{z}}{\partial z^{2}}=\rho_{c} \frac{\partial^{2} U_{z}}{\partial t^{2}} .
\end{array}
$$

The displacement vector can be written in terms of three scalar potentials $\phi, \chi$, and $\psi{ }^{16}$

$$
\mathbf{u}=\boldsymbol{\nabla} \phi+\nabla \times\left(\chi \hat{e}_{z}\right)+a \boldsymbol{\nabla} \times \boldsymbol{\nabla} \times\left(\psi \hat{e}_{z}\right),
$$

where $a$ is the radius of the cylinder which is a constant with dimensions of length. Substituting Eq. (11) in Eqs. (8)-(10) will result in the following set of equations:

$$
\begin{aligned}
& \left(\nabla^{2}-\frac{\partial^{2}}{\partial z^{2}}\right)\left\{c_{11} \nabla^{2} \phi+\left(c_{13}+2 c_{44}-c_{11}\right) \frac{\partial^{2} \phi}{\partial z^{2}}-\rho_{c} \frac{\partial^{2} \phi}{\partial t^{2}}\right. \\
& \quad+a \frac{\partial}{\partial z}\left[\left(c_{11}-c_{13}-c_{44}\right) \nabla^{2} \psi+\left(c_{13}+2 c_{44}-c_{11}\right) \frac{\partial^{2} \psi}{\partial z^{2}}\right. \\
& \left.\left.\quad-\rho_{c} \frac{\partial^{2} \psi}{\partial t^{2}}\right]\right\}=0 \\
& \frac{\partial}{\partial z}\left[\left(c_{13}+2 c_{44}\right) \nabla^{2} \phi+\left(c_{33}-c_{13}-2 c_{44}\right) \frac{\partial^{2} \phi}{\partial z^{2}}-\rho_{c} \frac{\partial^{2} \phi}{\partial t^{2}}\right] \\
& \quad+a\left(\frac{\partial^{2}}{\partial z^{2}}-\nabla^{2}\right)\left[c_{44} \nabla^{2} \psi+\left(c_{33}-c_{13}-2 c_{44}\right) \frac{\partial^{2} \psi}{\partial z^{2}}\right. \\
& \left.\quad-\rho_{c} \frac{\partial^{2} \psi}{\partial t^{2}}\right]=0
\end{aligned}
$$




$$
\begin{aligned}
\left(\nabla^{2}-\frac{\partial^{2}}{\partial z^{2}}\right)\left[\frac{\left(c_{11}-c_{12}\right)}{2} \nabla^{2} \chi+\left(c_{44}-\frac{\left(c_{11}-c_{12}\right)}{2}\right) \frac{\partial^{2} \chi}{\partial z^{2}}\right. \\
\left.-\rho_{c} \frac{\partial^{2} \chi}{\partial t^{2}}\right]=0 .
\end{aligned}
$$

The first two equations, Eqs. (12) and (13), represent the $P$ (compressional) and $S V$ (vertically polarized shear) waves. The compressional wave, represented by $\phi$, and the $S V$ wave, represented by $\psi$, are coupled. As can be seen in Eq. (14), the $S H$ (horizontally polarized shear) wave, represented by $\chi$, is decoupled. Equations (12)-(14) should be solved for $\phi, \psi$, and $\chi$. The normal mode expansion method is used, assuming solutions of the form:

$$
\begin{aligned}
& \phi=\sum_{n=0}^{\infty} B_{n} J_{n}(s r) \cos n \theta e^{i\left(k_{z} z-\omega t\right)}, \\
& \psi=\sum_{n=0}^{\infty} C_{n} J_{n}(s r) \cos n \theta e^{i\left(k_{z} z-\omega t\right)}, \\
& \chi=\sum_{n=0}^{\infty} D_{n} J_{n}(s r) \sin n \theta e^{i\left(k_{z} z-\omega t\right)} .
\end{aligned}
$$

Substituting Eqs. (15) and (16) in Eqs. (12) and (13) gives

$$
\left[\begin{array}{cc}
-\left[-c_{11} s^{2}-\left(c_{13}+2 c_{44}\right) k_{z}^{2}+\rho_{c} \omega^{2}\right] s^{2} & -a i k_{z}\left[-\left(c_{11}-c_{13}-c_{44}\right) s^{2}-c_{44} k_{z}^{2}+\rho_{c} \omega^{2}\right] s^{2} \\
i k_{z}\left[-\left(c_{13}+2 c_{44}\right) s^{2}-c_{33} k_{z}^{2}+\rho_{c} \omega^{2}\right] & a s^{2}\left[-c_{44} s^{2}-\left(c_{33}-c_{13}-c_{44}\right) k_{z}^{2}+\rho_{c} \omega^{2}\right]
\end{array}\right]\left[\begin{array}{l}
B_{n} \\
C_{n}
\end{array}\right]=0 .
$$

For a nontrivial solution, the coefficient determinant in Eq. (18) must be zero. This yields the following characteristic equation:

$$
c_{11} c_{44} s^{4}-\xi s^{2}+\zeta=0,
$$

where

$$
\begin{aligned}
\xi= & \left(c_{13}+c_{44}\right)^{2} k_{z}^{2}+c_{11}\left(\rho_{c} \omega^{2}-c_{33} k_{z}^{2}\right) \\
& +c_{44}\left(\rho_{c} \omega^{2}-c_{44} k_{z}^{2}\right), \\
\zeta= & \left(\rho_{c} \omega^{2}-c_{44} k_{z}^{2}\right)\left(\rho_{c} \omega^{2}-c_{33} k_{z}^{2}\right) .
\end{aligned}
$$

There are two solutions, $s_{1}$ and $s_{2}$, for Eq. (19),

$$
\begin{aligned}
& s_{1}^{2}=\frac{\xi-\sqrt{\xi^{2}-4 \zeta c_{11} c_{44}}}{2 c_{11} c_{44}}, \\
& s_{2}^{2}=\frac{\xi+\sqrt{\xi^{2}-4 \zeta c_{11} c_{44}}}{2 c_{11} c_{44}},
\end{aligned}
$$

which implies that the potential functions should be of the form: ${ }^{9,12}$

$$
\begin{aligned}
& \phi=\sum_{n=0}^{\infty}\left[B_{n} J_{n}\left(s_{1} r\right)+q_{2} C_{n} J_{n}\left(s_{2} r\right)\right] \cos n \theta e^{i\left(k_{z} z-\omega t\right)}, \\
& \psi=\sum_{n=0}^{\infty}\left[q_{1} B_{n} J_{n}\left(s_{1} r\right)+C_{n} J_{n}\left(s_{2} r\right)\right] \cos n \theta e^{i\left(k_{z} z-\omega t\right)},
\end{aligned}
$$

where

$$
\begin{aligned}
& q_{1}=-\frac{-c_{11} s_{1}^{2}-\left(c_{13}+2 c_{44}\right) k_{z}^{2}+\rho_{c} \omega^{2}}{a i k_{z}\left[-\left(c_{11}-c_{13}-c_{44}\right) s_{1}^{2}-c_{44} k_{z}^{2}+\rho_{c} \omega^{2}\right]}, \\
& q_{2}=-\frac{a i k_{z}\left[-\left(c_{11}-c_{13}-c_{44}\right) s_{2}^{2}-c_{44} k_{z}^{2}+\rho_{c} \omega^{2}\right]}{-c_{11} s_{2}^{2}-\left(c_{13}+2 c_{44}\right) k_{z}^{2}+\rho_{c} \omega^{2}} .
\end{aligned}
$$

It can be easily shown that for the potential function $\chi$ corresponding to the $S H$ wave,

$$
s_{3}^{2}=\frac{2\left(\rho_{c} \omega^{2}-c_{44} k_{z}^{2}\right)}{c_{11}-c_{12}},
$$

and therefore,

$$
\chi=\sum_{n=0}^{\infty} D_{n} J_{n}\left(s_{3} r\right) \sin n \theta e^{i\left(k_{z} z-\omega t\right)} .
$$

For an isotropic cylinder the $c_{i j}$ values can be written in terms of the Lamé constants $\lambda$ and $\mu$,

$$
c_{11}=c_{33}=\lambda+2 \mu, \quad c_{12}=c_{13}=\lambda, \quad c_{44}=\mu .
$$

It can be easily shown that by substituting the above values into Eqs. (24), (25), and (29), these equations are reduced to

$$
\begin{aligned}
& \phi=\sum_{n=0}^{\infty} B_{n} J_{n}\left(k_{l} r\right) \cos n \theta e^{i\left(k_{z} z-\omega t\right)}, \\
& \psi=\sum_{n=0}^{\infty} C_{n} J_{n}\left(k_{t} r\right) \cos n \theta e^{i\left(k_{z} z-\omega t\right)}, \\
& \chi=\sum_{n=0}^{\infty} D_{n} J_{n}\left(k_{t} r\right) \sin n \theta e^{i\left(k_{z} z-\omega t\right)},
\end{aligned}
$$

where

$$
k_{l}=\left(\frac{\omega^{2}}{c_{l}^{2}}-k_{z}^{2}\right)^{1 / 2}, \quad k_{t}=\left(\frac{\omega^{2}}{c_{t}^{2}}-k_{z}^{2}\right)^{1 / 2} .
$$

Equations (31)-(33) are the corresponding potential functions for an isotropic cylinder.

The boundary conditions at $r=a \operatorname{are}^{4}$

$$
\begin{aligned}
& -\frac{1}{\rho} \frac{\partial}{\partial r}\left(p_{i}+p_{s}\right)=\frac{\partial^{2} U_{r}}{\partial t^{2}}, \quad \sigma_{r r}=-\left(p_{i}+p_{s}\right), \\
& \sigma_{r \theta}=0, \quad \sigma_{r z}=0,
\end{aligned}
$$

F. Honarvar and A. N. Sinclair: Scattering from cylinders 
TABLE I. Physical parameters.

\begin{tabular}{lccc}
\hline \hline & $\begin{array}{c}\rho \\
\left(\mathrm{kg} / \mathrm{m}^{3}\right)\end{array}$ & $\begin{array}{c}c_{L} \\
(\mathrm{~m} / \mathrm{s})\end{array}$ & $\begin{array}{c}c_{T} \\
(\mathrm{~m} / \mathrm{s})\end{array}$ \\
\hline Aluminum & 2690 & 6420 & 3040 \\
Water & 1000 & 1475 & \\
\hline \hline
\end{tabular}

where $\rho$ is the density of the fluid surrounding the cylinder. Expanded expressions for displacements and stresses in terms of potential functions are given in the Appendix. By inserting the potential functions from Eqs. (24), (25), and (29) in Eq. (35), a system of four linear algebraic equations is obtained for each value of $n$ :

$$
\left(\begin{array}{cccc}
a_{11} & a_{12} & a_{13} & a_{14} \\
a_{21} & a_{22} & a_{23} & a_{24} \\
0 & a_{32} & a_{33} & a_{34} \\
0 & a_{42} & a_{43} & a_{44}
\end{array}\right)\left(\begin{array}{c}
A_{n} \\
B_{n} \\
C_{n} \\
D_{n}
\end{array}\right)=\left(\begin{array}{c}
b_{1} \\
b_{2} \\
0 \\
0
\end{array}\right) .
$$

Elements $a_{i j}$ and $b_{i}$ of the matrices in Eq. (36) are given in the Appendix. Equation (36) can be solved for $A_{n}$ for any specified frequency and position angle $\theta$ using Kramer's rule. The usual approach is to solve the problem in the far field $(r \gg a$ ) at a specific angle $\theta$ for a range of frequencies. The resulting far-field backscattered amplitude spectrum, which is called the form function, is obtained from the following equation: ${ }^{17}$

$$
\left|f_{\infty}\right|=\left(\frac{2 r}{a}\right)^{1 / 2}\left(\frac{p_{s}}{p_{i}}\right) e^{-i k r} .
$$

\section{RESULTS AND DISCUSSION}

To verify the mathematical model, it is initially applied to an isotropic aluminum cylinder. Acoustic properties of the cylinder and water are given in Table I. ${ }^{18}$ Noting that for an isotropic material,

$$
c_{L}=\sqrt{\frac{\lambda+2 \mu}{\rho}}, \quad c_{T}=\frac{\mu}{\rho},
$$

and using Eq. (30), the five elastic constants $c_{i j}$ for the isotropic aluminum cylinder can be found to be

$$
\begin{aligned}
& c_{11}=c_{33}=1.10872 \times 10^{11} \mathrm{~N} / \mathrm{m}^{2}, \\
& c_{12}=c_{13}=6.11523 \times 10^{10} \mathrm{~N} / \mathrm{m}^{2}, \\
& c_{44}=2.48599 \times 10^{10} \mathrm{~N} / \mathrm{m}^{2},
\end{aligned}
$$

where it is noted that there are only two independent constants due to the condition of isotropy. Using the above values, the form function of the aluminum cylinder is calculated for four different values of $\alpha$, see Fig. 2. The resulting form functions are identical to those in Fig. 2 of Ref. 18, thereby verifying that the model works for isotropic cylinders and generates the same results as the simple mathematical model used for isotropic cylinders. In these numerical calculations and those which follow, the number of normal modes, $N$, used in evaluating the series is equal to $N=k a_{\max }+5$, where $k a_{\max }$ is the maximum value of the normalized frequency $k a$ on the graph. This number of normal modes guarantees that
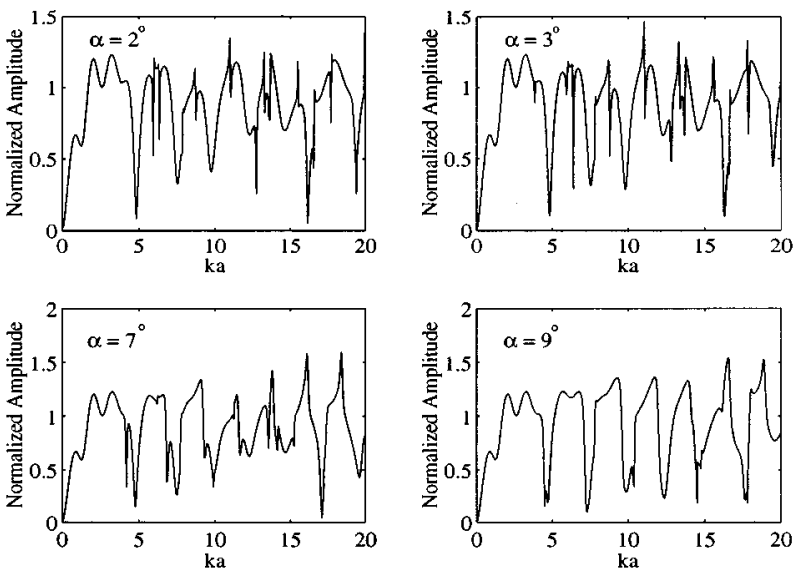

FIG. 2. Form functions for aluminum cylinder.

for the desired range of frequencies, the form function is correct (Ref. 4, p. 245).

To further investigate the mathematical model, elastic constants of the aluminum cylinder are perturbed and the resulting effects on the form functions are studied. What is expected is that by varying $c_{33}$, which characterizes the material along the cylinder axis, only the resonances associated with guided waves ${ }^{19}$ would be affected. Although the dependency of guided waves on the value of $c_{44}$ is not as obvious as their dependency on $c_{33}$, perturbations of $c_{44}$ are expected to show a similar effect on the resonant frequencies of the guided modes. This is because $c_{44}$ links the stresses and strains in the $r-z$ and $\theta-z$ planes for a transversely isotropic material. If guided waves which travel along the axis are of a shear type, they are either generated in the $r-z$ or the $\theta-z$ plane, which means they will be disturbed by perturbations in $c_{44}$. Variations of $c_{11}$ and $c_{12}$, which characterize the cylinder on the transverse plane, should mostly affect the resonances associated with Rayleigh and Whispering Gallery (WG) waves. Variations of $c_{13}$, which links stresses and strains along all three principal axes, should affect all three types of resonances.

In all the calculations that follow, the angle of incidence is taken to be equal to $3^{\circ}$. First $c_{33}$ and $c_{44}$ are perturbed. A $5 \%$ increase in $c_{44}$ shifts all resonances associated with guided waves to the right while Rayleigh and WG resonances remain almost unaffected, see Fig. 3. In Fig. 3(a), each resonance is designated by two integers. The first integer is the mode number $n$, and the second one is the mode type, designated by either $l$ or $p$. For resonances associated with Rayleigh waves $l=1$ and for those associated with WG waves $l \geqslant 2$. Values of $p$ correspond to axially guided waves and are underlined so that they can be distinguished from $l$ values. The effect of a perturbation in $c_{44}$ can be seen more clearly on Regge pole trajectories in Fig. 3(b). Each pole is designated by a " + " sign and corresponds to one of the resonant frequencies of Fig. 3(a).

Doubling the value of $c_{33}$ has a similar effect to that of perturbing $c_{44}$, as shown in Fig. 4 . While the sensitivity of the resonances to perturbations in these two elastic constants are different, $5 \%$ for $c_{44}$ and $100 \%$ for $c_{33}$, the general effect is the same. An increase in the value of each of these param- 

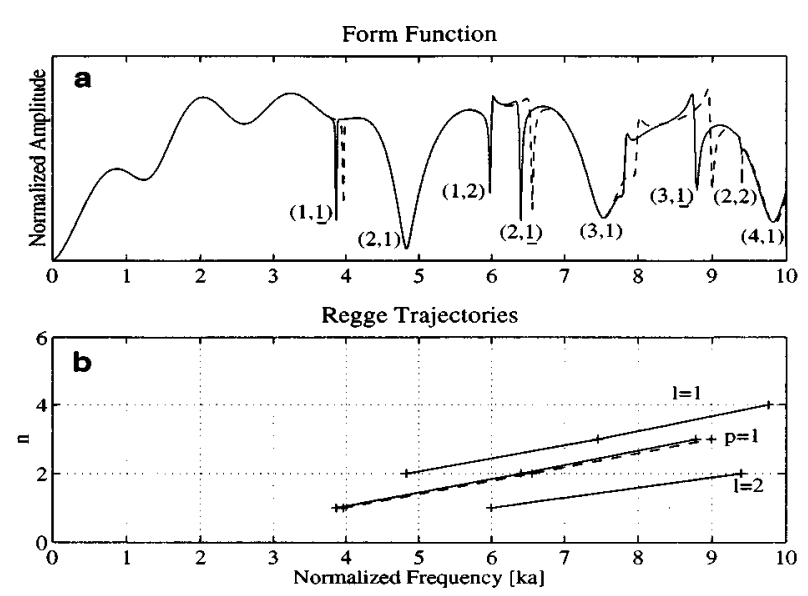

FIG. 3. Aluminum cylinder insonified by an infinite plane acoustic wave at an angle of incidence $\alpha=3^{\circ}$. (a) Form function. (b) Regge trajectories. Solid lines correspond to isotropic material properties as given in Eq. (40) and dashed lines illustrate the resulting changes in the form function if the value of $c_{44}$ is increased by $5 \%$.

eters produces a significant shift in resonant frequencies associated with guided waves, as predicted. The relatively high sensitivity of guided waves to perturbations of $c_{44}$ indicates that these waves are mostly of a shear type.

Next the values of $c_{11}$ and $c_{12}$ are each increased by $4 \%$. The resulting form functions are shown in Figs. 5 and 6, respectively. In Fig. 5 all Rayleigh and WG resonances are shifted to the right while resonances associated with guided waves remain unchanged. Similar behavior is observed in Fig. 6, but with the shift of resonances to the left. Again these results are as expected. Since Rayleigh and WG waves propagate mostly on the transverse plane, in particular for small angles of incidence $\alpha$, these waves are significantly affected by variations of $c_{11}$ and $c_{12}$. On the other hand, guided waves which propagate along the cylinder axis are hardly affected by perturbations of $c_{11}$ and $c_{12}$.

The form function resulting from increasing the value of $c_{13}$ by a factor of 2.5 is shown in Fig. 7(a). In this case, all
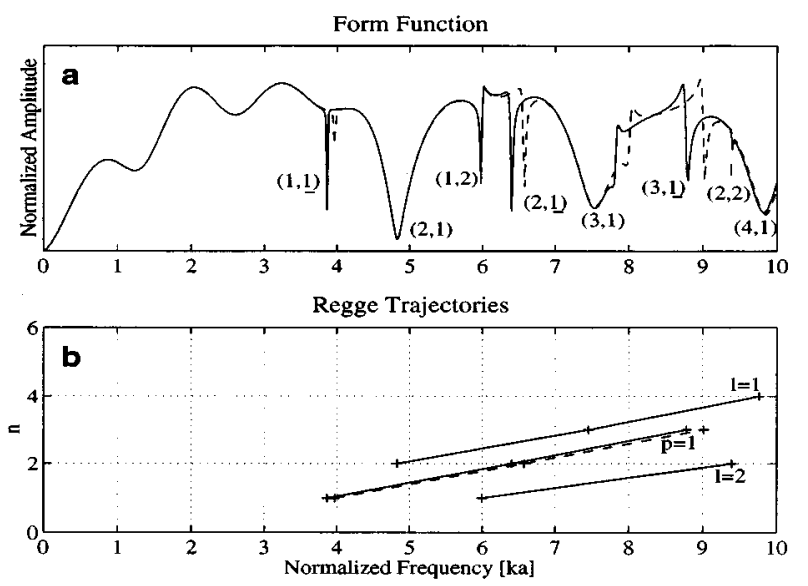

FIG. 4. Aluminum cylinder insonified by an infinite plane acoustic wave at an angle of incidence $\alpha=3^{\circ}$. (a) Form function. (b) Regge trajectories. Solid lines correspond to isotropic material properties as given in Eq. (40) and dashed lines illustrate the resulting changes in the form function if the value of $c_{33}$ is doubled.
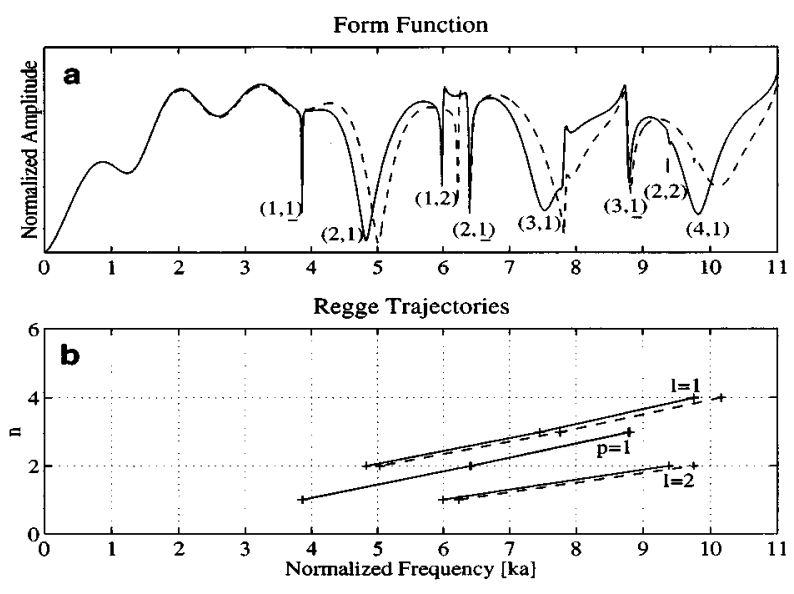

FIG. 5. Aluminum cylinder insonified by an infinite plane acoustic wave at an angle of incidence $\alpha=3^{\circ}$. (a) Form function. (b) Regge trajectories. Solid lines correspond to isotropic material properties as given in Eq. (40) and dashed lines illustrate the resulting changes in the form function if the value of $c_{11}$ is increased by $4 \%$.

three types of resonances, i.e., Rayleigh, WG, and guided wave modes, are affected. Rayleigh resonances are the least sensitive to changes in $c_{13}$ while guided waves show the largest shift. This result also agrees with the earlier predictions.

\section{SUMMARY AND CONCLUSIONS}

A mathematical model for acoustic wave scattering from a transversely isotropic cylinder is developed. Numerical calculations for isotropic cylinders yield identical results to those achieved with traditional simple models. The validity of the new formulation in modeling an anisotropic cylinder is investigated by perturbing the elastic constants of an isotropic aluminum cylinder. As expected, variations of $c_{11}$ and $c_{12}$, which characterize the cylinder on the transverse plane, affect the Rayleigh and WG resonances while guided waves, which propagate along the cylinder axis, remain unaffected. Conversely, changes in $c_{33}$ and $c_{44}$, which characterize the
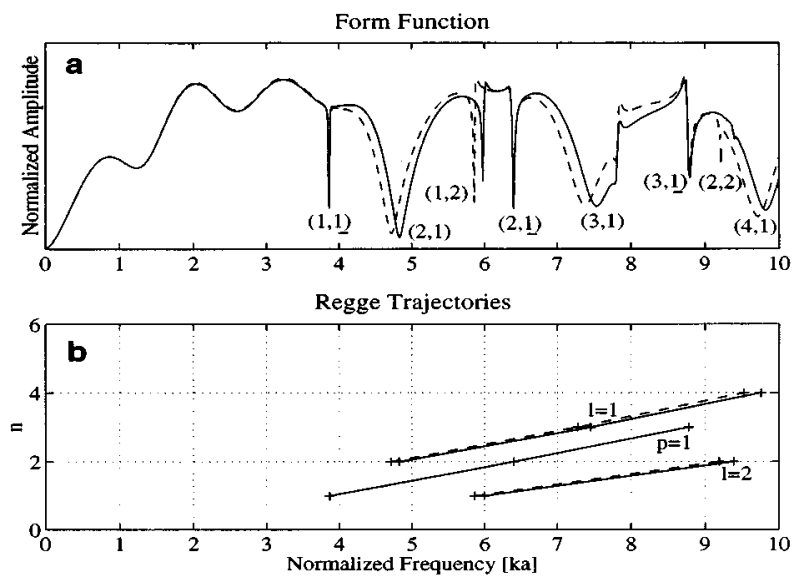

FIG. 6. Aluminum cylinder insonified by an infinite plane acoustic wave at an angle of incidence $\alpha=3^{\circ}$. (a) Form function. (b) Regge trajectories. Solid lines correspond to isotropic material properties as given in Eq. (40) and dashed lines illustrate the resulting changes in the form function if the value of $c_{12}$ is increased by $4 \%$. 

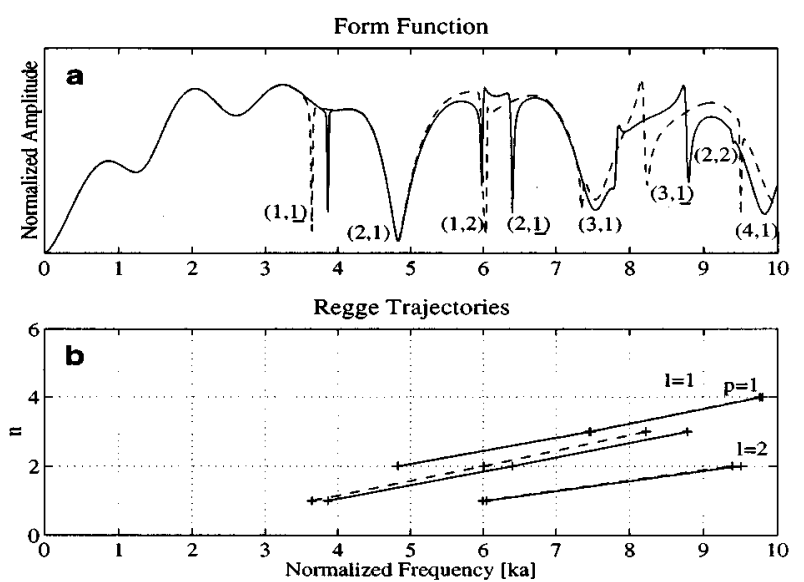

FIG. 7. Aluminum cylinder insonified by an infinite plane acoustic wave at an angle of incidence $\alpha=3^{\circ}$. (a) Form function. (b) Regge trajectories. Solid lines correspond to isotropic material properties as given in Eq. (40) and dashed lines illustrate the resulting changes in the form function if the value of $c_{13}$ is increased by a factor of 2.5 .

cylinder along the axis, cause the resonances associated with guided waves to be significantly affected while Rayleigh and WG resonances remain unchanged. Variations of $c_{13}$ affect all resonances, although axially guided modes appear to be particularly sensitive to values of this parameter. Further investigation is required in this area.

The mathematical model developed in this paper can be used in conjunction with experimental data for nondestructive testing of cylinders and on-line monitoring of the variations of mechanical properties of cylindrical products. Similar mathematical models could be developed for shells and multilayered cylinders.

\section{ACKNOWLEDGMENTS}

This work was supported by a grant from the Manufacturing Research Corporation of Ontario, and the Natural Sciences and Engineering Research Council (NSERC) of Canada.

\section{APPENDIX}

Expanding the displacements, Eq. (11), and stresses, Eq. (4), in terms of potential functions gives

$$
\begin{aligned}
U_{r} & =\frac{\partial \phi}{\partial r}+\frac{1}{r} \frac{\partial \chi}{\partial \theta}+a \frac{\partial^{2} \psi}{\partial r \partial z}, \\
U_{\theta} & =\frac{1}{r} \frac{\partial \phi}{\partial \theta}-\frac{\partial \chi}{\partial r}+\frac{a}{r} \frac{\partial^{2} \psi}{\partial \theta \partial z}, \\
U_{z} & =\frac{\partial \phi}{\partial z}-a\left(\frac{\partial^{2} \psi}{\partial r^{2}}+\frac{1}{r} \frac{\partial \psi}{\partial r}+\frac{1}{r^{2}} \frac{\partial^{2} \psi}{\partial \theta^{2}}\right), \\
\sigma_{r r} & =c_{11} \epsilon_{r r}+c_{12} \epsilon_{\theta \theta}+c_{13} \epsilon_{z z} \\
& =c_{11}\left(\frac{\partial U_{r}}{\partial r}\right)+c_{12}\left(\frac{U_{r}}{r}+\frac{1}{r} \frac{\partial U_{\theta}}{\partial \theta}\right)+c_{13} \frac{\partial U_{z}}{\partial z}
\end{aligned}
$$

$$
\begin{aligned}
& =c_{11}\left(\frac{\partial^{2} \phi}{\partial r^{2}}+\frac{1}{r} \frac{\partial^{2} \chi}{\partial \theta \partial r}-\frac{1}{r^{2}} \frac{\partial \chi}{\partial \theta}+a \frac{\partial^{3} \psi}{\partial z \partial r^{2}}\right) \\
& +c_{12}\left(\frac{1}{r} \frac{\partial \phi}{\partial r}+\frac{1}{r^{2}} \frac{\partial \chi}{\partial \theta}+\frac{a}{r} \frac{\partial^{2} \psi}{\partial z \partial r}+\frac{1}{r^{2}} \frac{\partial^{2} \phi}{\partial \theta^{2}}\right. \\
& \left.-\frac{1}{r} \frac{\partial^{2} \chi}{\partial \theta \partial r}+\frac{a}{r^{2}} \frac{\partial^{3} \psi}{\partial z \partial \theta^{2}}\right)+c_{13}\left[\frac{\partial^{2} \phi}{\partial z^{2}}-a\left(\frac{\partial^{3} \psi}{\partial z \partial r^{2}}\right.\right. \\
& \left.\left.+\frac{1}{r} \frac{\partial^{2} \psi}{\partial z \partial r}+\frac{1}{r^{2}} \frac{\partial^{3} \psi}{\partial z \partial \theta^{2}}\right)\right] \text {, } \\
& \sigma_{r \theta}=\frac{\left(c_{11}-c_{12}\right)}{2}\left(2 \epsilon_{r \theta}\right) \\
& =\frac{\left(c_{11}-c_{12}\right)}{2}\left(\frac{\partial U_{\theta}}{\partial r}-\frac{U_{\theta}}{r}+\frac{1}{r} \frac{\partial U_{r}}{\partial \theta}\right) \\
& =\frac{\left(c_{11}-c_{12}\right)}{2}\left(-\frac{2}{r^{2}} \frac{\partial \phi}{\partial \theta}+\frac{2}{r} \frac{\partial^{2} \phi}{\partial \theta \partial r}-\frac{\partial^{2} \chi}{\partial r^{2}}\right. \\
& \left.-\frac{2 a}{r^{2}} \frac{\partial^{2} \psi}{\partial z \partial \theta}+\frac{2 a}{r} \frac{\partial^{3} \psi}{\partial z \partial \theta \partial r}+\frac{1}{r} \frac{\partial \chi}{\partial r}+\frac{1}{r^{2}} \frac{\partial^{2} \chi}{\partial \theta^{2}}\right), \\
& \sigma_{r z}=c_{44}\left(2 \epsilon_{r z}\right) \\
& =c_{44}\left(\frac{\partial U_{z}}{\partial r}+\frac{\partial U_{r}}{\partial z}\right) \\
& =c_{44}\left[2 \frac{\partial^{2} \phi}{\partial z \partial r}-a\left(\frac{\partial^{3} \psi}{\partial r^{3}}+\frac{1}{r} \frac{\partial^{2} \psi}{\partial r^{2}}-\frac{1}{r^{2}} \frac{\partial \psi}{\partial r}\right.\right. \\
& \left.\left.+\frac{1}{r^{2}} \frac{\partial^{3} \psi}{\partial \theta^{2} \partial r}-\frac{2}{r^{3}} \frac{\partial^{2} \psi}{\partial \theta^{2}}-\frac{\partial^{3} \psi}{\partial z^{2} \partial r}\right)+\frac{1}{r} \frac{\partial^{2} \chi}{\partial z \partial \theta}\right] .
\end{aligned}
$$

Elements of the matrices given in Eq. (36) are as follows:

$$
\begin{aligned}
a_{11}= & \frac{P_{0} i^{n} \epsilon_{n}\left(k_{z} a\right) H_{n}^{\prime}\left(k_{z} a\right)}{\rho \omega^{2}}, \\
a_{12}= & \left(1+i q_{1} k_{z}\right)\left[n J_{n}\left(s_{1} a\right)-s_{1} a J_{n+1}\left(s_{1} a\right)\right], \\
a_{13}= & \left(q_{2}+i k_{z}\right)\left[n J_{n}\left(s_{2} a\right)-s_{2} a J_{n+1}\left(s_{2} a\right)\right], \\
a_{14}= & J_{n}\left(s_{3} a\right) n, \\
a_{21}= & P_{0} i^{n} \epsilon_{n} H_{n}^{\prime}\left(k_{z} a\right), \\
a_{22}= & {\left[c_{11}+i\left(c_{11}-c_{13}\right) q_{1} k_{z}\right]\left[\left(n^{2}-n-s_{1}^{2} a^{2}\right) J_{n}\left(s_{1} a\right)\right.} \\
& \left.+s_{1} a J_{n+1}\left(s_{1} a\right)\right]+\left[c_{12}+\left(c_{12}-c_{13}\right) i q_{1} k_{z}\right] \\
& \times\left[n J_{n}\left(s_{1} a\right)-s_{1} a J_{n+1}\left(s_{1} a\right)\right]+\left[-c_{13} k_{z}^{2}-c_{12} n^{2}\right. \\
& \left.+\left(c_{13}-c_{12}\right) i n^{2} q_{1} k_{z}\right] J_{n}\left(s_{1} a\right), \\
a_{23}= & {\left[c_{11} q_{2}+i\left(c_{11}-c_{13}\right) k_{z}\right]\left[\left(n^{2}-n-s_{2}^{2} a^{2}\right) J_{n}\left(s_{2} a\right)\right.} \\
& \left.+s_{2} a J_{n+1}\left(s_{2} a\right)\right]+\left[c_{12} q_{2}+\left(c_{12}-c_{13}\right) i k_{z}\right] \\
& \times\left[n J_{n}\left(s_{2} a\right)-s_{2} a J_{n+1}\left(s_{2} a\right)\right]+\left[\left(-c_{13} k_{z}^{2}\right.\right. \\
& \left.\left.-c_{12} n^{2}\right) q_{2}+\left(c_{13}-c_{12}\right) i n^{2} k_{z}\right] J_{n}\left(s_{2} a\right),
\end{aligned}
$$

F. Honarvar and A. N. Sinclair: Scattering from cylinders 


$$
\begin{aligned}
a_{24}= & \left(c_{11}-c_{12}\right) n\left[(n-1) J_{n}\left(s_{3} a\right)-s_{3} a J_{n+1}\left(s_{3} a\right)\right], \\
a_{31}= & 0, \\
a_{32}= & 2 n\left(1+i q_{1} k_{z}\right)\left[(1-n) J_{n}\left(s_{1} a\right)+s_{1} a J_{n+1}\left(s_{1} a\right)\right], \\
a_{33}= & 2 n\left(q_{2}+i k_{z}\right)\left[(1-n) J_{n}\left(s_{2} a\right)+s_{2} a J_{n+1}\left(s_{2} a\right)\right], \\
a_{34}= & {\left[s_{3}^{2} a^{2}-2 n(n-1)\right] J_{n}\left(s_{3} a\right)-2 s_{3} a J_{n+1}\left(s_{3} a\right), } \\
a_{41}= & 0, \\
a_{42}= & {\left[q_{1}\left(s_{1}^{2} a^{2}-k_{z}^{2}\right)+2 i k_{z}\right]\left[n J_{n}\left(s_{1} a\right)\right.} \\
& \left.-s_{1} a J_{n+1}\left(s_{1} a\right)\right], \\
a_{43}= & \left(s_{2}^{2} a^{2}-k_{z}^{2}+2 i k_{z} q_{2}\right)\left[n J_{n}\left(s_{2} a\right)-s_{2} a J_{n+1}\left(s_{2} a\right)\right], \\
a_{44}= & i n k_{z} J_{n}\left(s_{3} a\right), \\
b_{1}= & \frac{a P_{0} i^{n} \epsilon_{n}\left(k_{z} a\right) J_{n}^{\prime}\left(k_{z} a\right)}{\rho \omega^{2}}, \\
b_{2}= & -P_{0} i^{n} \epsilon_{n} a^{2} J_{n}\left(k_{z} a\right) .
\end{aligned}
$$

${ }^{1}$ J. J. Faran, Jr., J. Acoust. Soc. Am. 23, 405 (1951).

${ }^{2}$ L. Flax, V. K. Varadan, and V. V. Varadan, J. Acoust. Soc. Am. 68, 1832 (1980).
${ }^{3}$ F. Léon, F. Lecroq, D. Décultot, and G. Maze, J. Acoust. Soc. Am. 91, 1388 (1992).

${ }^{4}$ N. D. Veksler, Resonance Acoustic Spectroscopy, Springer Series on Wave Phenomena (Springer-Verlag, Berlin, 1993).

${ }^{5}$ I. Mirsky, J. Acoust. Soc. Am. 37, 1016 (1965).

${ }^{6}$ I. Mirsky, J. Acoust. Soc. Am. 37, 1022 (1965).

${ }^{7}$ Y. M. Tsai, S. N. Liu, and G. M. Light, "Cylindrically guided waves in a transversely isotropic shaft,"' in Review of Progress in Quantitative Nondestructive Testing, edited by D. O. Thompson and D. E. Chimenti (Plenum, New York, 1990), Vol. 9, pp. 251-255.

${ }^{8}$ Y. M. Tsai, J. Pressure Vessel Technol. 113, 585 (1991).

${ }^{9}$ J. E. White and C. Tongtaow, J. Acoust. Soc. Am. 70, 1147 (1981).

${ }^{10}$ A. K. Chan and L. Tsang, J. Acoust. Soc. Am. 74, 1605 (1983).

${ }^{11}$ T. Kundu and A. Boström, J. Appl. Mech. 58, 695 (1991).

${ }^{12}$ B. Zhang, H. Dong, and K. Wang, J. Acoust. Soc. Am. 96, 2546 (1994).

${ }^{13}$ M. de Billy, J. Acoust. Soc. Am. 97, 852 (1995).

${ }^{14}$ M. de Billy, Acustica 81, 281 (1995).

${ }^{15}$ L. E. Malvern, Introduction to the Mechanics of a Continuous Medium (Prentice-Hall, Englewood Cliffs, NJ, 1969), p. 668.

${ }^{16} \mathrm{P}$. M. Morse and H. Feshbach, Methods of Theoretical Physics (McGrawHill, New York, 1953), pp. 1764-1767.

${ }^{17}$ L. Flax, G. C. Gaunaurd, and H. Überall, "Theory of Resonance Scattering," in Physical Acoustics, edited by W. P. Mason and R. N. Thurston (Academic, New York, 1981), Vol. 15, Chap. 3, pp. 191-294.

${ }^{18}$ T. B. Li and M. Ueda, J. Acoust. Soc. Am. 86, 2363 (1989).

${ }^{19}$ G. Maze, J. L. Izbicki, and J. Ripoche, J. Acoust. Soc. Am. 77, 1352 (1985). 